\title{
Correlation and Path Analysis for Yield and Yield Components in Blackgram [Vigna mungo (L.) Hepper]
}

\author{
Ranjeet A. Tambe*, Gabrial M. Lal, and Pramod W. Ramteke
}

Department of Genetics and Plant Breeding, Naini Agriculture Institute, Sam Higginbottom University of Agriculture, Technology and Sciences, Allahabad-211007 (U.P.), India.

*Corresponding author

\section{Keywords \\ Black gram [Vignamungo (L.) Hepper], Genetic variability, correlation, Path analysis}

Article Info

Accepted: 15 June 2018 Available Online: 10 July 2018

\section{A B S T R A C T}

The experimental material was consisting of 41 Black gram genotypes, check as T-9, during kharif 2017. The experiment was laid out in Randomised Complete Block Design with 3 replications at field experimentation centre of Department of Genetics and Plant Breeding, Sam Higginbottom University of Agriculture, Technology \& Sciences. The observations were logged on five randomly taken plants to each treatment and replication for 13 quantitative characters viz. days to $50 \%$ flowering, days to $50 \%$ pod setting, plant height, number of primary branches per plant, clusters per plant, pods per plant, pod length, seeds per pod, days to maturity, seed index, biological yield, harvest index and seed yield to estimate the variability, heritability and genetic advance as $\%$ mean, character association and path analysis. High heritability along with high Genetic advance as \% mean was observed for harvest index and seed yield per plant represents simple selection is effective to improve these characters. The correlations revealed that harvest index, seeds per pod, days to $50 \%$ pod setting, pods per plant, days to $50 \%$ flowering, seed index and biological yield have the significant positive association with the seed yield per plant at both genotypic and phenotypic levels. The path analysis revealed that the harvest index, biological yield, days to $50 \%$ flowering, plant height, pod length and clusters per plant had shown the true relationship with seed yield by establishing the positive correlations and direct effects at both genotypic and phenotypic levels, while branches per plant and days to maturity at genotypic levels and pods per plant and seeds per pod at phenotypic levels.

\section{Introduction}

Blackgram [Vigna mungo (L.) Hepper], Chromosome number $2 \mathrm{n}=22$, is a selfpollinating and widely cultivated grain legume. It is one of the most important pulse crops grown in India. The cultivated blackgram belongs to the family Leguminosae, sub-family Papilionaceae. It is mainly a day neutral warm season crop commonly grown in semi-arid to sub-humid low land tropics and sub-tropics. This crop is grown in cropping systems as a mixed crop, cash crop, sequential crop besides growing as 
sole crop under residual moisture conditions after the harvest of rice and also before and after the harvest of other summer crops under semi irrigated and dry land conditions (Parveen et al., 2011).

Variability refers to the presence of differences among the individuals of plant population.Itresultsduetodifferenceeitherinthe geneticconstitutionoftheindividualof a population or the environment they have grown. The existence of variability is essential for improvement of genetic material. The study of genetic variability in any crop would help in the genetic improvement of yield and desirable characters. It will facilitate the identification of proper genotypes for a particular agro-climate. Identification, characterization and study of genotypes and genetic homology between them would provideabaseforfurtherstudiesforcropimprove ment.Theobservedphenotypicvariation is the result of an interaction between genotype and environment in which the individuals are grown. However, it is only genetic variation which is heritable and hence important in any selection programme.

Grain yield is complex character, which depends on its main components viz; number of pod per plant, pod length, number of seed per pod and 100 seed weight. These components are further dependent for their expression on several morphological and developmental traits, which are interrelated with each other and therefore, the parent selected for the breeding programmes aimed at increased seed yield should possess wide range of genetic variation for the above said morphological and developmental characters. Besides, it could be of interest to know the magnitude of variation due to heritable component, which in turn would be a guide for selection for the improvement of a population. In other words, for the improvement in any crop species, the knowledge of genetic variability for characters of economic importance and their heritability and genetic advance is of utmost importance in planning future breeding programme (Singh et al., 2007).

Seed yield is a complex trait and is influenced by number of component traits. The study on inter-relationship between the component traits and seed yield will formulate an effective and viable breeding programme for improvement of yield in a short time. Studies on correlation values indicate the intensity and direction of association of a character with yield. Path analysis identifies the yield components with direct and indirect influence on the yield. Hence, the present research work was undertaken to assess the correlation and path coefficients estimates of economically important plant characteristics and to determine the characteristics contributing to seed yield in blackgram (Patidar and Sharma, 2017).

In view of these facts, 41 blackgram genotypes were evaluated in this study to estimate genetic variability, correlation coefficient and direct and indirect effect of yield and yield components on grain yield to screen out the suitable genotype for exploitation in a breeding programme aimed at improving grain yield potential of blackgram.

\section{Materials and Methods}

The present investigation was carried out at the Field Experimentation Centre, Department of Genetics and Plant Breeding, Naini Agricultural Institute, Sam Higginbottom University of Agriculture, Technology and Sciences, Allahabad, U.P. (India) during Kharif-2017.The experimental materials constituted of the germplasm collection of 41 genotypes of Black gram [Vigna mungo (L) Hepper], procured from Department of Agriculture Botany, Dr.Punjabrao Deshmukh 
Krishi Vidyapeeth, Akola, (Maharashtra). Data were recorded from five randomly selected plants from each genotype per replication and the average was taken for analysis. All the recommended package of practices was followed to raise a good crop. The experiment was laid out in Randomised Complete Block Design with 3 replications. The genotypes were sown by hand dibbling in each plot by imposing randomisation in each replication along with check T-9. Each plot has 4 rows with the spacing of row to row $30 \mathrm{~cm}$ and plant to plant $10 \mathrm{~cm}$. Standard statistical procedures were used for the analysis of correlation coefficient values(r) at genotypic and phenotypic levels by Johnson et al., (1955) and described by Singh and Choudhary (1985).

Path coefficient analysis was utilized to partition the phenotypic and genotypic correlation coefficient into the direct effects and indirect effects along with residual effects. The analysis was carried out as per the equation suggested by Dewey and Lu (1959) originally proposed by Wright (1921) and described by Singh and Choudhary (1985).

\section{Results and Discussion}

The analysis of variance revealed highly significant to significant differences among the genotypes for all the thirteen characters studied (Table 1). In the present study, variation among the characters is estimated by Genotypic Coefficient of Variation (GCV) and Phenotypic Coefficient of Variation (PCV). The PCV was higher than the GCV for few characters indicates the interaction of genotypes with the environment (Table 2). High GCV and PCV were recorded for harvest index (20.52 and 21.86) followed by seed yield /plant (18.67 and 19.89), clusters per plant (15.40 and 17.12) and biological yield (14.26 and 14.43).
Estimates of heritability are a good index for predicting the transmission of characters from parents to their offspring (Falconer, 1981). High heritability (broad sense) was recorded for characters i.e., biological yield per plant (97.68\%), followed by days to $50 \%$ flowering (95.75\%), days to $50 \%$ pod setting (94.66\%), plant height $(94.55 \%)$,pods per plant $(93.76 \%)$, harvest index $(88.15 \%)$, seeds per plant $(88.11 \%)$. High heritability alone may not lead to valid conclusions unless it is accompanied with the Genetic advance as percent mean (Johnson and Robinson, 1955). High heritability coupled with high genetic advance as percent of the mean was recorded for harvest index, seed yield per plant and biological yield. These findings are in accordance with Rajashekhar et al., (2017) and Rolaniya et al., (2017).

The genotypic and phenotypic correlation coefficients were computed among 13 characters (Table 3). The correlations revealed that harvest index, seeds per pod,days to $50 \%$ pod setting, pods per plant, days to $50 \%$ flowering, seed index and biological yield have the significant positive association with the seed yield per plant at both genotypic and phenotypic levels, while pod length and plant height showing negative but significant association with seed yield at both genotypic as well as phenotypic level. Similar result found Babu et al., (2016) Therefore, these characters appeared as greatest important associates of seed yield per plant and have also been observed by preceding workers (Sushmitharaj et al., 2018; Hemalatha et al., 2017, Hemavathy et al., 2015).

The correlation values provided only nature and degree of relationship of yield contributing characters on seed yield. Path coefficient analysis is a statistical technique to split the observed correlation coefficients into direct and indirect effects of independent variables on the dependent variable. In the 
present study, path coefficient analysis was carried out using genotypic and phenotypic correlation matrix of 13 characters (table 5). The path analysis revealed that the harvest index, biological yield, days to $50 \%$ flowering, plant height, pod length and clusters per plant had shown the true relationship with seed yield by establishing the positive correlations and direct effects at both genotypic and phenotypic levels, while branches per plant and days to maturity at genotypic levels and pods per plant and seeds per pod at phenotypic levels. These results were in accordance with the findings of Bharti et al., (2013), Kanimoli et al., (2015) and Patidar and Sharma (2017). By considering the nature and extent of correlation coefficients and their direct and indirect effects it can be concluded that improvement of Black gram seed yield is brought through simultaneous selection seeds per pod,pod per plant, biological yield and harvest index.

Table.1 Analysis of variance for different characters of Black gram

\begin{tabular}{|l|l|c|c|c|}
\hline S. No. & Parameters & \multicolumn{3}{|c|}{ Mean Sum of Squares } \\
\cline { 3 - 5 } & & $\begin{array}{c}\text { Replications } \\
(\mathbf{d f}=\mathbf{2})\end{array}$ & $\begin{array}{c}\text { Genotypes } \\
(\mathbf{d f}=\mathbf{4 0})\end{array}$ & $\begin{array}{c}\text { Error } \\
(\mathbf{d f}=\mathbf{8 0})\end{array}$ \\
\hline $\mathbf{1}$ & Days to 50\% Flowering & 0.1707 & $40.9000^{* *}$ & 0.5957 \\
\hline $\mathbf{2}$ & Days to 50\% Pod Setting & 0.1545 & $42.7073^{* *}$ & 0.7878 \\
\hline $\mathbf{3}$ & Plant Height (cm) & 0.1729 & $102.4780^{* *}$ & 1.9295 \\
\hline $\mathbf{4}$ & Branches/ Plant & 0.4315 & $0.2271^{*}$ & 0.1469 \\
\hline $\mathbf{5}$ & Clusters/ Plant & 0.0315 & $5.7850^{* *}$ & 0.4205 \\
\hline $\mathbf{6}$ & Pods/ Plant & 0.0452 & $33.2665^{* *}$ & 0.7215 \\
\hline $\mathbf{7}$ & Pod Length (cm) & 0.2518 & $0.3487^{* *}$ & 0.0820 \\
\hline $\mathbf{8}$ & Seeds/ Pod & 0.0296 & $0.7776^{* *}$ & 0.3023 \\
\hline $\mathbf{9}$ & Days to Maturity & 2.2520 & $27.8175^{* *}$ & 1.3187 \\
\hline $\mathbf{1 0}$ & Seed Index (g) & 0.1642 & $1.2499^{* *}$ & 0.0600 \\
\hline $\mathbf{1 1}$ & Seed Yield/ Plant (g) & 0.1876 & $8.1714^{* *}$ & 0.3517 \\
\hline $\mathbf{1 2}$ & Biological Yield (g) & 1.7044 & $191.2677^{* *}$ & 1.4959 \\
\hline $\mathbf{1 3}$ & Harvest Index (\%) & 0.2681 & $32.8512^{* *}$ & 1.4083 \\
\hline
\end{tabular}

$* \& * *$ Significant at $5 \% \& 1 \%$ level of significant respectively 
Table.2 Genetic parameter of different characters in Blackgram

\begin{tabular}{|c|c|c|c|c|c|c|c|c|}
\hline S.NO & Character & VG & VP & $\begin{array}{l}\text { GCV } \\
(\%)\end{array}$ & $\begin{array}{l}\text { PCV } \\
(\%)\end{array}$ & $\begin{array}{l}h^{2} b s \\
\%\end{array}$ & GA & GA as $\%$ mean \\
\hline 1 & Days to $50 \%$ Flowering & 13.43 & 14.03 & 9.65 & 9.86 & 95.75 & 7.39 & 19.44 \\
\hline 2 & Days to $50 \%$ Pod Setting & 13.97 & 14.76 & 7.65 & 7.86 & 94.66 & 7.49 & 15.32 \\
\hline 3 & Plant Height $(\mathrm{cm})$ & 33.52 & 35.45 & 10.70 & 11.01 & 94.55 & 11.60 & 21.44 \\
\hline 4 & Branches/ Plant & 0.03 & 0.17 & 6.75 & 17.20 & 15.39 & 0.13 & 5.45 \\
\hline 5 & Clusters/ Plant & 1.79 & 2.21 & 15.40 & 17.12 & 80.95 & 2.48 & 28.55 \\
\hline 6 & Pods/ Plant & 10.85 & 11.57 & 9.86 & 10.19 & 93.76 & 6.57 & 19.67 \\
\hline 7 & Pod Length $(\mathrm{cm})$ & 0.09 & 0.17 & 6.83 & 9.46 & 52.02 & 0.44 & 10.14 \\
\hline 8 & Seeds/ Pod & 0.16 & 0.46 & 7.89 & 13.45 & 34.39 & 0.48 & 9.53 \\
\hline 9 & Days to Maturity & 8.83 & 10.15 & 4.38 & 4.70 & 87.00 & 5.71 & 8.42 \\
\hline 10 & Seed Index (g) & 0.40 & 0.46 & 12.72 & 13.65 & 87.86 & 1.21 & 24.43 \\
\hline 11 & Seed Yield/ Plant (g) & 2.61 & 2.96 & 18.67 & 19.89 & 88.11 & 3.12 & 36.10 \\
\hline 12 & Biological Yield (g) & 63.26 & 64.75 & 14.26 & 14.43 & 97.68 & 16.19 & 29.04 \\
\hline 13 & Harvest Index (\%) & 10.48 & 11.89 & 20.52 & 21.86 & 88.15 & 6.26 & 39.69 \\
\hline
\end{tabular}


Table.3 Correlation coefficient between yield and its related traits in 41Blackgram genotypes at Genotypic level

\begin{tabular}{|c|c|c|c|c|c|c|c|c|c|c|c|c|c|c|}
\hline No & Character & $\begin{array}{l}\text { Days to } \\
50 \% \\
\text { Flowerin }\end{array}$ & $\begin{array}{l}\text { Days to } \\
50 \% \text { Pod } \\
\text { Setting }\end{array}$ & $\begin{array}{l}\text { Plant } \\
\text { Height }\end{array}$ & $\begin{array}{l}\text { Branches/ } \\
\text { Plant }\end{array}$ & $\begin{array}{l}\text { Clusters/ } \\
\text { Plant }\end{array}$ & $\begin{array}{l}\text { Pods/ } \\
\text { Plant }\end{array}$ & $\begin{array}{l}\text { Pod } \\
\text { Length }\end{array}$ & $\begin{array}{l}\text { Seeds/ } \\
\text { Pod }\end{array}$ & $\begin{array}{l}\text { Days to } \\
\text { Maturity }\end{array}$ & $\begin{array}{l}\text { Seed } \\
\text { Index }\end{array}$ & $\begin{array}{l}\text { Biological } \\
\text { Yield }\end{array}$ & $\begin{array}{l}\text { harvest } \\
\text { Index }\end{array}$ & $\begin{array}{l}\text { Seed } \\
\text { Yield/ } \\
\text { Plant }\end{array}$ \\
\hline $\mathbf{1}$ & Days to $50 \%$ Flowering & 1.00 & $0.933 * *$ & -0.073 & $-0.298 * *$ & $-0.157 *$ & -0.012 & $-0.472 * *$ & -0.084 & $0.753 * *$ & $0.580 * *$ & -0.103 & $0.293 * *$ & $0.285^{* *}$ \\
\hline 2 & $\begin{array}{l}\text { Days to } 50 \% \text { Pod } \\
\text { Setting }\end{array}$ & & 1.00 & $-0.164 *$ & -0.118 & $-0.174 *$ & 0.055 & $-0.415^{* *}$ & 0.002 & $0.798 * *$ & $0.572 * *$ & -0.120 & $0.382 * *$ & $0.359 * *$ \\
\hline 3 & Plant Height & & & 1.00 & 0.089 & $-0.182 *$ & $-0.267 * *$ & $0.249 * *$ & 0.021 & 0.013 & 0.152 & 0.133 & $-0.275^{* *}$ & $-0.201^{*}$ \\
\hline 4 & Branches/ Plant & & & & 1.00 & 0.092 & 0.026 & $0.424 * *$ & $0.253 * *$ & -0.104 & -0.064 & 0.137 & -0.102 & -0.023 \\
\hline 5 & Clusters/ Plant & & & & & 1.00 & $0.443^{* *}$ & $0.165^{*}$ & $0.169 *$ & -0.108 & $-0.274 * *$ & -0.079 & $0.222 *$ & $0.207^{*}$ \\
\hline 6 & Pods/ Plant & & & & & & 1.00 & 0.031 & $-0.414 * *$ & -0.062 & -0.044 & $0.191 *$ & 0.152 & $0.345^{* *}$ \\
\hline 7 & Pod Length & & & & & & & 1.00 & $-0.221 *$ & -0.105 & $-0.165^{*}$ & 0.014 & $-0.332 * *$ & $-0.365^{* *}$ \\
\hline 8 & Seeds/ Pod & & & & & & & & 1.00 & 0.004 & $-0.190^{*}$ & -0.041 & $0.519 * *$ & $0.572 * *$ \\
\hline 9 & Days to Maturity & & & & & & & & & 1.00 & $0.494 * *$ & $-0.159^{*}$ & $0.207^{*}$ & 0.133 \\
\hline 10 & Seed Index & & & & & & & & & & 1.00 & -0.029 & $0.272 * *$ & $0.261 * *$ \\
\hline 11 & Biological Yield & & & & & & & & & & & 1.00 & $-0.528 * *$ & $0.196^{*}$ \\
\hline 12 & Harvest Index & & & & & & & & & & & & 1.00 & $0.723^{* *}$ \\
\hline 13 & Seed Yield/ Plant & & & & & & & & & & & & & 1.00 \\
\hline
\end{tabular}

$* \& *$ Significant at $1 \%$ and $5 \%$ level of significance respectively 
Table.4 Correlation coefficient between yield and its related traits in 41blackgram genotypes at phenotypic level

\begin{tabular}{|c|c|c|c|c|c|c|c|c|c|c|c|c|c|c|}
\hline $\begin{array}{l}\dot{z} \\
\dot{i n}\end{array}$ & 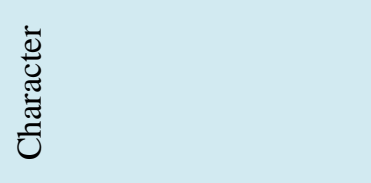 & 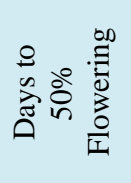 & 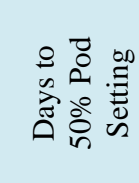 & 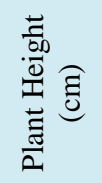 & 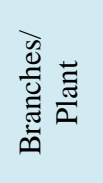 & 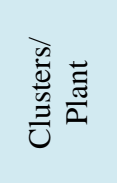 & 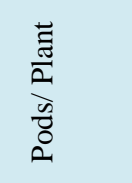 & 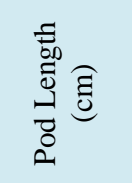 & $\begin{array}{l}\overrightarrow{0} \\
0 \\
\bar{z} \\
\dot{y} \\
\tilde{n}\end{array}$ & 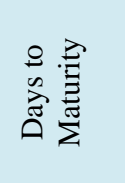 & 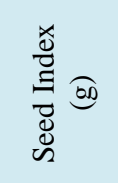 & 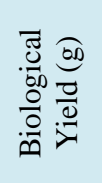 & 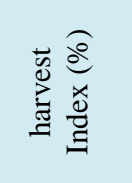 & 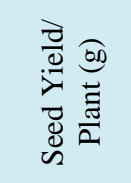 \\
\hline 1 & Days to $50 \%$ Flowering & 1.00 & $0.888^{* *}$ & -0.073 & -0.098 & -0.133 & -0.017 & $-0.337 * *$ & -0.012 & $0.689 * *$ & $0.514 * *$ & -0.107 & $0.266^{* *}$ & $0.254^{* *}$ \\
\hline 2 & Days to $50 \%$ Pod Setting & & 1.00 & -0.147 & -0.052 & -0.152 & 0.060 & $-0.283^{* * *}$ & -0.024 & $0.730^{* *}$ & $0.536 * *$ & -0.111 & $0.347^{* *}$ & $0.331^{* *}$ \\
\hline 3 & Plant Height (cm) & & & 1.00 & 0.042 & $-0.169 *$ & $-0.238^{* *}$ & $0.186^{*}$ & 0.031 & 0.020 & 0.144 & 0.130 & $-0.240 * *$ & $-0.172^{*}$ \\
\hline 4 & Branches/ Plant & & & & 1.00 & 0.057 & -0.008 & $0.159^{*}$ & -0.008 & -0.063 & -0.007 & 0.047 & -0.016 & 0.001 \\
\hline 5 & Clusters/ Plant & & & & & 1.00 & $0.403 * *$ & 0.122 & 0.057 & -0.077 & $-0.220 *$ & -0.061 & 0.134 & 0.126 \\
\hline 6 & Pods/ Plant & & & & & & 1.00 & 0.001 & $-0.266 * *$ & -0.048 & -0.024 & $0.188^{*}$ & 0.137 & $0.315^{* *}$ \\
\hline 7 & Pod Length $(\mathrm{cm})$ & & & & & & & 1.00 & -0.078 & -0.083 & -0.089 & 0.028 & $-0.242 * *$ & $-0.249 * *$ \\
\hline 8 & Seeds/ Pod & & & & & & & & 1.00 & 0.001 & -0.144 & -0.029 & $0.310^{* *}$ & $0.335^{* *}$ \\
\hline 9 & Days to Maturity & & & & & & & & & 1.00 & $0.429 * *$ & -0.142 & $0.176^{*}$ & 0.112 \\
\hline 10 & Seed Index (g) & & & & & & & & & & 1.00 & -0.018 & $0.237^{* *}$ & $0.233 * *$ \\
\hline 11 & Biological Yield (g) & & & & & & & & & & & 1.00 & $-0.505 * *$ & $0.183^{*}$ \\
\hline 12 & harvest Index (\%) & & & & & & & & & & & & 1.00 & $0.748^{* *}$ \\
\hline 13 & Seed Yield/ Plant (g) & & & & & & & & & & & & & 1.00 \\
\hline
\end{tabular}

$* \& *$ Significant at $1 \%$ and $5 \%$ level of significance respectively 
Table.5 Direct and indirect effects between yield and its related traits in 41Blackgram genotypes at genotypic level

\begin{tabular}{|c|c|c|c|c|c|c|c|c|c|c|c|c|c|c|}
\hline ż & 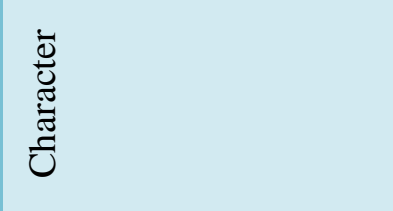 & 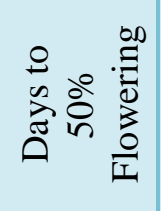 & 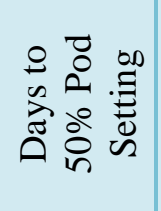 & 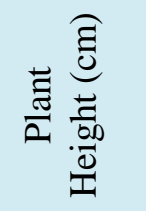 & 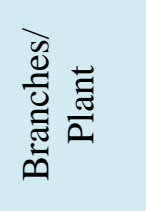 & 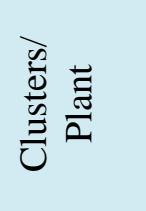 & $\begin{array}{l}\frac{\vec{\Xi}}{a} \\
\frac{\vec{n}}{0} \\
0\end{array}$ & 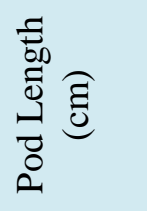 & $\begin{array}{l}\overrightarrow{0} \\
2 \\
\bar{z} \\
\bar{d} \\
\tilde{n}\end{array}$ & 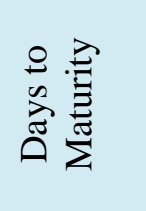 & 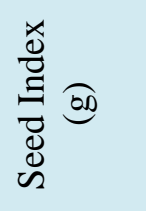 & 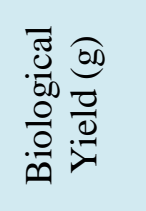 & 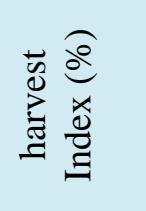 & 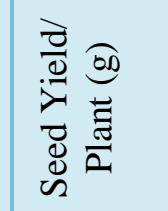 \\
\hline 1 & Days to $50 \%$ Flowering & 0.1989 & 0.1856 & -0.0145 & -0.0593 & -0.0312 & -0.0024 & -0.0939 & -0.0167 & 0.1499 & 0.1154 & -0.0206 & 0.0583 & $0.2849 * *$ \\
\hline 2 & $\begin{array}{l}\text { Days to } 50 \% \text { Pod } \\
\text { Setting }\end{array}$ & -0.1647 & -0.1764 & 0.0288 & 0.0208 & 0.0308 & -0.0098 & 0.0732 & -0.0004 & -0.1408 & -0.1010 & 0.0211 & -0.0674 & $0.3586 * *$ \\
\hline 3 & Plant Height $(\mathrm{cm})$ & -0.0022 & -0.0050 & $\mathbf{0 . 0 3 0 7}$ & 0.0027 & -0.0056 & -0.0082 & 0.0076 & 0.0007 & 0.0004 & 0.0047 & 0.0041 & -0.0084 & $-0.2006^{*}$ \\
\hline 4 & Branches/ Plant & -0.0163 & -0.0065 & 0.0049 & 0.0547 & 0.0051 & 0.0014 & 0.0232 & 0.0138 & -0.0057 & -0.0035 & 0.0075 & -0.0056 & -0.0227 \\
\hline 5 & Clusters/ Plant & -0.0003 & -0.0003 & -0.0003 & 0.0002 & 0.0018 & 0.0008 & 0.0003 & 0.0003 & -0.0002 & -0.0005 & -0.0001 & 0.0004 & $0.2072 *$ \\
\hline 6 & Pods/ Plant & 0.0008 & -0.0038 & 0.0183 & -0.0018 & -0.0304 & -0.0687 & -0.0022 & 0.0284 & 0.0043 & 0.0030 & -0.0131 & -0.0104 & $0.3448 * *$ \\
\hline 7 & Pod Length $(\mathrm{cm})$ & -0.0036 & -0.0032 & 0.0019 & 0.0033 & 0.0013 & 0.0002 & 0.0077 & -0.0017 & -0.0008 & -0.0013 & 0.0001 & -0.0025 & $-0.3653 * *$ \\
\hline 8 & Seeds/ Pod & 0.0112 & -0.0003 & -0.0029 & -0.0338 & -0.0226 & 0.0553 & 0.0296 & -0.1337 & -0.0005 & 0.0254 & 0.0055 & -0.0694 & $0.5723 * *$ \\
\hline 9 & Days to Maturity & 0.0516 & 0.0547 & 0.0009 & -0.0072 & -0.0074 & -0.0043 & -0.0072 & 0.0003 & 0.0685 & 0.0338 & -0.0109 & 0.0142 & 0.1328 \\
\hline 10 & Seed Index (g) & -0.0873 & -0.0861 & -0.0229 & 0.0096 & 0.0412 & 0.0067 & 0.0247 & 0.0286 & -0.0742 & -0.1504 & 0.0043 & -0.0408 & $0.2611 * *$ \\
\hline 11 & Biological Yield (g) & -0.0930 & -0.1076 & 0.1200 & 0.1230 & -0.0709 & 0.1721 & 0.0128 & -0.0370 & -0.1427 & -0.0257 & 0.8994 & -0.4747 & $0.1958 *$ \\
\hline 12 & harvest Index (\%) & 0.3897 & 0.5076 & -0.3656 & -0.1350 & 0.2953 & 0.2016 & -0.4412 & 0.6898 & 0.2747 & 0.3610 & -0.7015 & 1.3291 & $0.7227 * *$ \\
\hline
\end{tabular}
Bold are direct effects, R SQUARE $=0.9898$, RESIDUAL EFFECT $=0.1012$. 
Table.6 Direct and indirect effects between yield and its related traits in 41Blackgram genotypes at phenotypic level

\begin{tabular}{|c|c|c|c|c|c|c|c|c|c|c|c|c|c|c|}
\hline No & Character & $\begin{array}{l}\text { Days to } \\
50 \% \\
\text { Flowering }\end{array}$ & $\begin{array}{l}\text { Days to } \\
50 \% \\
\text { Pod } \\
\text { Setting }\end{array}$ & $\begin{array}{l}\text { Plant } \\
\text { Height } \\
(\mathrm{cm})\end{array}$ & $\begin{array}{l}\text { Branche } \\
\text { s/ Plant }\end{array}$ & $\begin{array}{l}\text { Clusters } \\
\text { / Plant }\end{array}$ & $\begin{array}{l}\text { Pods/ } \\
\text { Plant }\end{array}$ & $\begin{array}{l}\text { Pod } \\
\text { Length } \\
(\mathrm{cm})\end{array}$ & $\begin{array}{l}\text { Seeds/ } \\
\text { Pod }\end{array}$ & $\begin{array}{l}\text { Days to } \\
\text { Maturity }\end{array}$ & $\begin{array}{l}\text { Seed } \\
\text { Index } \\
(\mathrm{g})\end{array}$ & $\begin{array}{l}\text { Biologic } \\
\text { al Yield } \\
(\mathrm{g})\end{array}$ & $\begin{array}{l}\text { harvest } \\
\text { Index } \\
(\%)\end{array}$ & $\begin{array}{l}\text { Seed } \\
\text { Yield/ } \\
\text { Plant (g) }\end{array}$ \\
\hline $\mathbf{1}$ & $\begin{array}{l}\text { Days to } 50 \% \\
\text { Flowering }\end{array}$ & 0.0834 & 0.0741 & -0.0061 & -0.0082 & -0.0111 & -0.0014 & -0.0281 & -0.0010 & 0.0575 & 0.0428 & -0.0089 & 0.0222 & $0.2538 * *$ \\
\hline 2 & $\begin{array}{l}\text { Days to } 50 \% \text { Pod } \\
\text { Setting }\end{array}$ & -0.0101 & -0.0114 & 0.0017 & 0.0006 & 0.0017 & -0.0007 & 0.0032 & 0.0003 & -0.0083 & -0.0061 & 0.0013 & -0.0040 & $0.3311^{* *}$ \\
\hline 3 & Plant Height $(\mathrm{cm})$ & -0.0012 & -0.0023 & 0.0157 & 0.0007 & -0.0027 & -0.0037 & 0.0029 & 0.0005 & 0.0003 & 0.0023 & 0.0020 & -0.0038 & $-0.1721 *$ \\
\hline 4 & Branches/ Plant & 0.0012 & 0.0007 & -0.0005 & -0.0127 & -0.0007 & 0.0001 & -0.0020 & 0.0001 & 0.0008 & 0.0001 & -0.0006 & 0.0002 & 0.0014 \\
\hline 5 & Clusters/ Plant & -0.0014 & -0.0016 & -0.0018 & 0.0006 & 0.0108 & 0.0043 & 0.0013 & 0.0006 & -0.0008 & -0.0024 & -0.0007 & 0.0015 & 0.1261 \\
\hline 6 & Pods/ Plant & -0.0004 & 0.0013 & -0.0052 & -0.0002 & 0.0088 & 0.0220 & 0.0000 & -0.0059 & -0.0011 & -0.0005 & 0.0041 & 0.0030 & $0.3146 * *$ \\
\hline 7 & Pod Length $(\mathrm{cm})$ & -0.0068 & -0.0057 & 0.0037 & 0.0032 & 0.0024 & 0.0000 & 0.0200 & -0.0016 & -0.0017 & -0.0018 & 0.0006 & -0.0048 & $-0.2492 * *$ \\
\hline 8 & Seeds/ Pod & -0.0001 & -0.0002 & 0.0002 & -0.0001 & 0.0004 & -0.0021 & -0.0006 & 0.0078 & 0.0000 & -0.0011 & -0.0002 & 0.0024 & $0.3346^{* *}$ \\
\hline 9 & Days to Maturity & -0.0027 & -0.0029 & -0.0001 & 0.0002 & 0.0003 & 0.0002 & 0.0003 & 0.0000 & -0.0039 & -0.0017 & 0.0006 & -0.0007 & 0.1115 \\
\hline 10 & Seed Index (g) & -0.0263 & -0.0274 & -0.0074 & 0.0004 & 0.0112 & 0.0012 & 0.0045 & 0.0074 & -0.0220 & -0.0511 & 0.0009 & -0.0121 & $0.2326 * *$ \\
\hline 11 & Biological Yield (g) & -0.0802 & -0.0833 & 0.0975 & 0.0350 & -0.0461 & 0.1409 & 0.0207 & -0.0218 & -0.1066 & -0.0136 & 0.7513 & -0.3794 & $0.1832 *$ \\
\hline 12 & harvest Index (\%) & 0.2983 & 0.3898 & -0.2698 & -0.0181 & 0.1509 & 0.1537 & -0.2715 & 0.3482 & 0.1973 & 0.2658 & -0.5672 & 1.1232 & $0.7477 * *$ \\
\hline
\end{tabular}




\section{References}

Al Jibouri, H.A., Mullar, P.A., and Rabinsion, H.F. (1958) Genetic and environment al variances and co-variances in an upland cotton cross of inter specific origin. Agronomy, 50:633-636.

Babu, J. S., Reni, Y. P. and Ramana, M.V. (2016) Character correlation and path coefficient in black gram [VignaMungo

Hepper].International Research Journal of Natural and Applied Sciences, 3(7): 178-185.

Babu, J. S., Reni, Y. P. and Ramana, M.V. (2016) Character correlation and path coefficient in black gram [VignaMungo

(L.)

Hepper].International Research Journal of Natural and Applied Sciences,3(7): 178-185.

Bandi, H. R.K., Rao, K. N., Krishna, K. V and Srinivasulu, K (2018) Correlation and path-coefficient estimates of yield and yield component traits in rice fallow blackgram [Vignamungo (L.) Hepper]. International Journal of Current Microbiology and Applied Sciences7(3): 3304-3309.

Dewey, D.R. and Lu, K.H. (1959) Correlation and path coefficient analysis of components of crested wheat grass seed production. Agronomy Journal.,51:515-518.

Falconer, D.S. (1981) Introduction to Quantitative genetics, $3^{\text {rd }}$ ed. Longman, New York.Pp 340.

Hemalatha, K., Lal, S. S. and Lal G. M. (2017) Study on Genetic Variability and Correlation in Blackgram (Vignamungo (L.)Hepper) Journal of PharmacognosyandPhytochemistry, 6(4): 674-676.

Hemavathy, A. T., Shunmugavalli, N and Anand, G. (2015) Genetic variability, correlation and path co-efficient studies on yield and its components in mungbean [Vignaradiata (L.) Wilezek].Legume Research,38(4): 442-446.

Johnson, H.W., Robinson, H.F. and Comstock.R.E. (1955) Genotypic and Phenotypic Correlations in Soybean and their implications in selection Agronomy.47: 477-438.

Parveen, S. I., Sekhar, M. R., Reddy, D. M. and Sudhakar, P. (2011) Correlation and path coefficient analysis for yield and yield components in blackgram (Vignamungo(1.) hepper). International Journal of Applied Biology and Pharmaceutical Technology.2(3): 619-625.

Patidar, M. and Sharma, H. (2017) Correlation and path coefficient studies in Blackgram (VignaMungo (L.) Hepper).Journal of Pharmacognosy and Phytochemistry,6(4): 1626-1628.

Rajasekhar, D., Lal, S. S. and Lal, G. M. (2017)Character association and path analysis for seed yield \& its components in black gram [vignamungo (L.) Hepper].Plant Archives, 17(1);467-471.

Rolaniya, D. K., Jinjwadiya, M. K., Meghawal, D. R and Lal, G.M. (2017) Studies on genetic variability in Black gram (Vignamungo L. Hepper) germplasm.Journal of Pharmacognosy and Phytochemistry, 6(4): 1506-1508.

Shivade, H. A. Rewale, A. P. and Patil, S. B. (2011) Correlation and path analysis for yield and yield components in black gram [Vignamungo (L.) Hepper].Legume Research; 34(3):178183.

Singh, I. P. Sanjay Kumar Singh, J. D. and Singh, K. P. (2007) Genetic variation, character association and path analysis between grain yield and its component in black gram [Vignamungo(L.) 
Hepper].Progressive

Agriculture 7(1/2):113-115.

Sushmitharaj, D. V., Shoba, D. and Pillai, M.

A. (2018) Genetic Variability and

Correlation Studies in Black Gram
(Vignamungo [L.] hepper) with Reference to YMV Resistance.International Journal of Current Microbiology and Applied Sciences6: 2849-2856.

\section{How to cite this article:}

Ranjeet A. Tambe, Gabrial M. Lal, and Pramod W. Ramteke. 2018. Correlation and Path Analysis for Yield and Yield Components in Blackgram [Vigna mungo (L.)Hepper] Int.J.Curr.Microbiol.App.Sci. 7(07): 2074-2084. doi: https://doi.org/10.20546/ijcmas.2018.707.244 